\title{
Displacement Immunosensor Based on Surface Plasmon Resonance for Rapid and Highly Sensitive Detection of 2,4,6-Trinitrotoluene
}

\author{
Takeshi Onodera*, Yutaka Mizuta ${ }^{1}$, Kazuhiko Horikawa ${ }^{1}$, Praveen Singh², \\ Kiyoshi Matsumoto ${ }^{3}$, Norio Miura ${ }^{4}$ and Kiyoshi Toko \\ Faculty of Information Science and Electrical Engineering, Kyushu University, \\ 744 Motooka, Nishi-ku, Fukuoka-shi, Fukuoka 819-0395, Japan \\ ${ }^{1}$ Graduate School of Information Science and Electrical Engineering, Kyushu University, \\ 744 Motooka, Nishi-ku, Fukuoka-shi, Fukuoka 819-0395, Japan \\ ${ }^{2}$ Division of Biophysics, Indian Veterinary Research Institute, Izatnagar 243 122, U.P., India \\ ${ }^{3}$ Faculty of Agriculture, Kyushu University, \\ 6-10-1 Hakozaki, Higashi-ku, Fukuoka-shi, Fukuoka 812-8581, Japan \\ ${ }^{4}$ Art, Science and Technology Centre for Cooperative Research, Kyushu University, \\ 6-1 Kasuga-kouen, Kasuga-shi, Fukuoka 816-8580, Japan
}

(Received June 1, 2010; accepted August 24, 2010)

Key words: surface plasmon resonance, displacement, TNT, self-assembled monolayer, antigenantibody interaction

By making use of the antigen-antibody interaction and a surface plasmon resonance (SPR) sensor, we developed a measurement procedure of displacement immunoassay to rapidly detect 2,4,6-trinitrotoluene (TNT). In this procedure, TNT solutions were injected in $30 \mathrm{~s}$ at the end of flowing an anti-TNT antibody. Three kinds of sensor surfaces were modified with TNT analogues, namely, TNP-glycine, DNP-glycine, and DNP-acetic acid in a self-assembled monolayer containing ethylene glycol. We investigated which of the TNT analogues provided higher sensitivity using the displacement immunosensor. As a result, the limit of detection (LOD) of TNT was $0.4 \mathrm{ng} / \mathrm{mL}$ (ppb) when using the DNP-glycine-modified Au sensor surface with a oneminute flow of TNT solution. We concluded that the final TNT LOD was $0.9 \mathrm{ppb}$ on the basis of experiments using the three different DNP-glycine-modified sensor surfaces. The LOD was $0.7 \mathrm{ppb}$ when using the sensorgram slope $10 \mathrm{~s}$ after TNT injection. The displacement immunosensor can detect TNT at sub-ppb levels in $12 \mathrm{~s}$.

\section{Introduction}

Currently, explosive detection systems such as explosive detection dogs, metal detectors, and X-ray inspection apparatus are mainly used. These systems have

"Corresponding author: e-mail: onodera@ed.kyushu-u.ac.jp 
disadvantages in any given situation. Therefore, to overcome the drawbacks, a novel detection system for explosives with mobility, sensitivity, and reliability is required to supplement these types of detection systems. Hence, we developed a prototype portable explosive sensor combined with a surface plasmon resonance (SPR) analytical instrument that has antigen-antibody interaction detection capability. The SPR analytical instrument is a highly sensitive transducer, and an antibody is used as a highly selective detector. ${ }^{(1)}$ We also developed polyclonal and monoclonal antibodies against 2,4,6-trinitrololuene (TNT) and 2,4-dinitrotoluene (DNT). ${ }^{(2,3)}$ In addition, we developed three kinds of sensor surfaces that are highly sensitive for the detection of nitroaromatic compounds. ${ }^{(4-6)}$ However, the detection time was over $15 \mathrm{~min}$ in those studies, because we chose an indirect competitive assay format for highly sensitive detection of explosives. In the assay format, the antibody and antigen solutions are premixed and incubated for about 15 min before measurement. However, in an actual situation, such as when it is used in an airport, a short measurement time is required for usability.

In contrast, a displacement immunoassay in a continuous flow system does not require incubation. ${ }^{(7)}$ An antibody is immobilized on a membrane or a column, and a fluorescence-labeled antigen is bound to the immobilized antibody before the antigen solution is injected. An antigen is introduced over the antibody, and the fluorescencelabeled antigen displaces the introduced antigen. The fluorescence intensity is detected downstream of the flow line..$^{(8,9)}$ A displacement assay using a combination of a labeled antibody and an immobilized antigen has also been reported. ${ }^{(10)}$ However, in these systems, the real-time measurement of the dissociation process has not been shown. Luminex (flow cytometer)-based displacement immunoassay has also been reported. ${ }^{(11)}$ That sensing system is not based on the flow system or the displacement reaction in the well on a microtiter plate, and 15 min of incubation time was required.

We have reported a displacement method using a polyclonal antibody and SPR analytical instrument for real-time measurement of 2,4,6-trinitrophenol (TNP), which is an explosive. (12) The displacement method with SPR recordes the dissociation period of the polyclonal antibody displaced by TNP from the sensor surface. However, the flow time of TNP is 5 min. We assume that the displacement method with our SPR sensor can be applied to measure solution extracted from a piece of cotton wiper after wiping a suspected part of explosive adhesive. About 1,000 ng TNT remains in a fingerprint as a signature of the explosive. ${ }^{(13)}$ The total detection time should be less than $60 \mathrm{~s}$ for practical use.

In this study, we developed the sensing procedure using the displacement method based on SPR for the rapid and highly sensitive detection of TNT. Our goal is to detect TNT with a ppb level of the limit of detection (LOD) and one-minute flow of TNT solution. We investigated the LOD of TNT by the displacement method for three kinds of sensor surfaces, which were fabricated using 2,4,6-trinitrophenyl glycine (TNP-gly), $N$-(2,4-dinitrophenyl) glycine (DNP-gly), and 2,4-dinitrophenyl acetic acid (DNP-aa) with self-assembled monolayers (SAMs). We also tried to detect TNT using the slope of the sensorgram $10 \mathrm{~s}$ after TNT injection to shorten the total detection time. 


\section{Materials and Methods}

\subsection{Reagents and chemicals}

The following chemicals were used without further purification: a mouse anti-TNT monoclonal antibody (TNT-Ab) was obtained from Strategic Biosolutions (Ramona, CA, USA). TNT solution (21.8 ppm) in Milli-Q water was purchased from Chugoku Kayaku Co., Ltd. (Hiroshima, Japan). TNP-gly was purchased from Research Organics (Cleveland, OH, USA). DNP-gly and DNP-aa were purchased from Tokyo Chemical Industry (Tokyo, Japan). The aromatic dialkanethiol PEG6-COOH was purchased from Senso Path Technologies (Bozeman, MT, USA). $N$-ethyl- $N$ '-(3-dimethylaminopropyl) carbodiimide (EDC) and $N$-hydroxysuccinimide (NHS) in an amine coupling kit (GE Healthcare Piscataway, NJ, USA) were used. All other chemicals were purchased either from Tokyo Chemical Industry, Japan, Wako Pure Chemical Industries, Inc., or Kanto Chemical, Co., Japan. All aqueous solutions were prepared using Milli-Q deionized water obtained from Milli-Q system (Millipore, Bedford, MA, USA).

\subsection{Fabrication of sensor surface}

A bare Au sensor chip in a SIA Au kit (GE Healthcare) was used in sensor chip fabrication. Sensor chips were fabricated by the procedure described in ref. 14 with slight modifications. After the cleaning of sensor chips and the SAMs formed on the sensor chips using PEG6-COOH, the SAM carboxyl terminal groups were converted to amino terminal groups by amine coupling with ethylenediamine. To immobilize the TNT analogues (DNP-gly, TNP-gly, and DNP-aa) by the amine coupling reaction, carboxyl groups of each analogue were activated with EDC and NHS solutions. Then the activated analogues were reacted with the amino-terminated SAM on the sensor chips.

\subsection{Instrument and conditions}

The interaction between the TNT analogue immobilized on the sensor surface and the antibody was analyzed with a BiacoreX system (GE Healthcare). This system is a typical SPR analytical instrument for the analysis of biomolecule interaction. ${ }^{(15)}$ All measurements were performed at $25^{\circ} \mathrm{C}$. HBS [10 mM HEPES-buffered saline, $150 \mathrm{mM}$ $\mathrm{NaCl}, 0.05 \%(\mathrm{v} / \mathrm{v})$ Tween 20, $\mathrm{pH} 7.4$ ] was used as the running buffer at a flow rate of 10 $\mu \mathrm{l} / \mathrm{min}$. To regenerate the sensor surface, $100 \mathrm{mM}$ sodium hydrate was run for $3 \mathrm{~s}$. TNT solutions of various concentrations were made by diluting TNT solution (Milli-Q water) with HBS.

\subsection{TNT detection by displacement immunoassay}

Premixing of the antibody and antigen solutions is not required before measurement by displacement immunoassay ${ }^{(16)}$ Figure 1 shows the principle of displacement immunoassay for detecting TNT in this study. When TNT-Ab is added to the TNT analogue immobilized on the sensor surface, the increase in SPR response is due to the binding of TNT-Ab to the immobilized TNT analogue. When the flow of TNT$\mathrm{Ab}$ stops, running buffer is automatically used, and the spontaneous dissociation of 


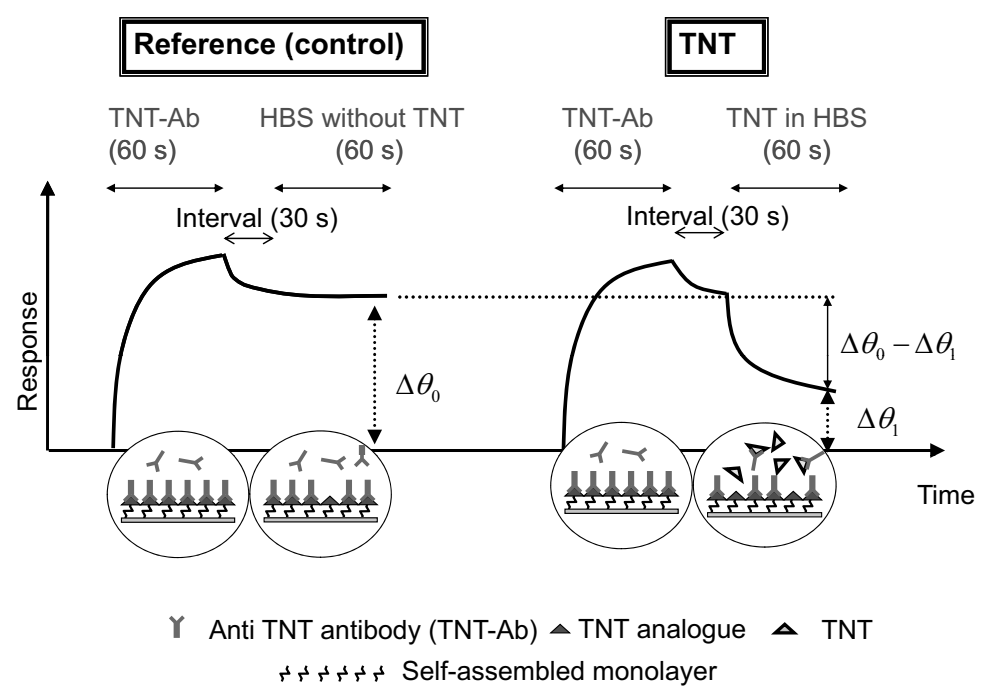

Fig. 1. Principle of displacement immunoassay using the SPR sensor.

the bound antibody starts immediately. Then, HBS without TNT or TNT solution in HBS is injected. HBS is used as a reference (control). When a high concentration of TNT molecules are present in the buffer solution, dissociation is accelerated and the sensor response decreases markedly. TNT-Ab bound to the TNT analogue immobilized on the sensor surface is bound to TNT instead of the TNT analogue. The TNT-AbTNT complex that is formed breaks away from the sensor surface. In this study, the displacement ratio was defined by

$$
\left(\Delta \theta_{0}-\Delta \theta_{1}\right) / \Delta \theta_{0} \cdot 100
$$

where $\Delta \theta_{0}$ (RU) and $\Delta \theta_{1}$ (RU) are the amounts of antibody remaining $10 \mathrm{~s}$ after adding HBS without TNT and with TNT solution, respectively. TNT solutions of various concentrations are allowed to flow in the displacement immunoassay. $\Delta \theta_{0}$ (RU) at $10 \mathrm{~s}$ after the flowing of the TNT solution or HBS was applied to eq. (1), and response curves were obtained.

After the flow of an injected solution, the sensor surface is washed using running buffer to remove nonspecific adsorption, in the normal configuration. However, in this experiment, the configuration was changed to "no washing" when TNT-Ab was injected, because the next sample could not be injected until washing was finished, which required about $1 \mathrm{~min}$, and spontaneous dissociation occurred during this time. Antibody concentrations at which the sensor surface was saturated with a 1 min injection were chosen: $25 \mu \mathrm{g} / \mathrm{mL}$ (ppm) for the DNP-gly-modified surface and $30 \mu \mathrm{g} / \mathrm{mL}$ for the TNPgly- and DNP-aa-modified surfaces. Thirty seconds after the end of flowing TNT-Ab, HBS (0, reference), and 10,100 pg/mL (ppt), and 1, $10 \mathrm{ng} / \mathrm{mL}$ (ppb) TNT solutions were allowed to flow in this order for $60 \mathrm{~s}$ at $10 \mu \mathrm{l} / \mathrm{min}$. This sequence was performed three 
times. $100 \mathrm{ng} / \mathrm{mL}$ (ppb) TNT solution was injected once in the final step, because TNT at high concentrations may adsorb onto the flow channel, and subsequent cycles cannot be measured correctly.

\section{Results and Discussion}

\subsection{Detection of TNT}

In displacement immunoassay using an immobilized antibody and a labeled antigen, signal intensity increases at a lower flow rate, because there is a longer interaction time between the antibody-labeled antigen complex and the antigen.(16,17) A higher sensitivity is also obtained using a combination of an immobilized antigen and a labeled antibody at a lower flow rate. ${ }^{(18)}$ Therefore, the flow rate was fixed at $10 \mu \mathrm{l} / \mathrm{min}$, which was lower than the default flow rate $(20 \mu \mathrm{l} / \mathrm{min})$ of BiacoreX.

A representative sensorgram obtained using the DNP-gly-immobilized sensor surface is shown in Fig. 2(a). The vertical axis indicates the sensor response in resonance units (RU). A resonance angle shift of $0.1^{\circ}$ was defined as $1,000 \mathrm{RU}$ (19) $^{(19}$ After injecting $25 \mu \mathrm{g} / \mathrm{mL}$ (25 ppm) antibody, there was a change in sensor response upon the binding of the antibody to the sensor surface, and the sensor response was almost saturated in $30 \mathrm{~s}$. After antibody flow, the sensor response gradually decreased owing to spontaneous dissociation. Spontaneous dissociation is observed in immunoassays using the SPR instrument (e.g., ref. 15), and this phenomenon has also been reported for the displacement immunoassay. ${ }^{(18)}$ TNT injection at 10 or $100 \mathrm{ng} / \mathrm{mL}$ caused a large decrease in response.

Figure 2(b) shows an overlay of sensorgrams shown in Fig. 2(a). Response curves for TNT flows of $0-100 \mathrm{pg} / \mathrm{mL}$ were the same and completely overlapped. However, the response curve for $1 \mathrm{ng} / \mathrm{mL}$ (ppb) TNT flow was clearly different from the $0-100 \mathrm{pg}$ / $\mathrm{mL}$ (ppt) curves, indicating that dissociation was promoted by as little as $1 \mathrm{ng} / \mathrm{mL}$ TNT. Response curves for $10 \mathrm{ng} / \mathrm{mL}$ (ppb) and $100 \mathrm{ng} / \mathrm{mL}(\mathrm{ppb}$ ) TNT indicated about 26\% and $66 \%$ displacements, respectively, compared with the response curve for injected HBS. In these cases, a large reduction was observed, and the flow of TNT solution was only 1 min. After the $100 \mathrm{ppb}$ TNT flow ended, the sensor response increased rapidly; this phenomenon was due to the minimal difference in refractive index between the running buffer and the solvent of $100 \mathrm{ng} / \mathrm{mL}$ (ppb) TNT solution.

The amount of bound antibody was $180 \mathrm{RU} 10 \mathrm{~s}$ before the injection of the TNT solution (or HBS) onto the DNP-aa-modified surface, and 430 RU was obtained for the TNP-gly-modified surface. In contrast, the amount of antibody bound onto the DNP-glymodified surface was 1,440 RU, and although this result was unclear, sensorgrams were probably saturated. The amounts of bound antibodies were almost the same for each measurement cycle (Fig. 2(b)), indicating that the sensor surface was stable and that a $3 \mathrm{~s}$ flow of $100 \mathrm{mM} \mathrm{NaOH}$ for regeneration yielded acceptable repeatability.

\subsection{Detection limit of TNT}

Figure 3 shows the displacement immunosensor response curves calculated using eq. (1) with $\Delta \theta_{0}$ and $\Delta \theta_{1}$ obtained on the DNP-gly-, DNP-aa-, and TNP-gly-modified surfaces. In the range of $10-100 \mathrm{ng} / \mathrm{mL}$ (ppb) TNT, the DNP-aa-modified surface displacement 


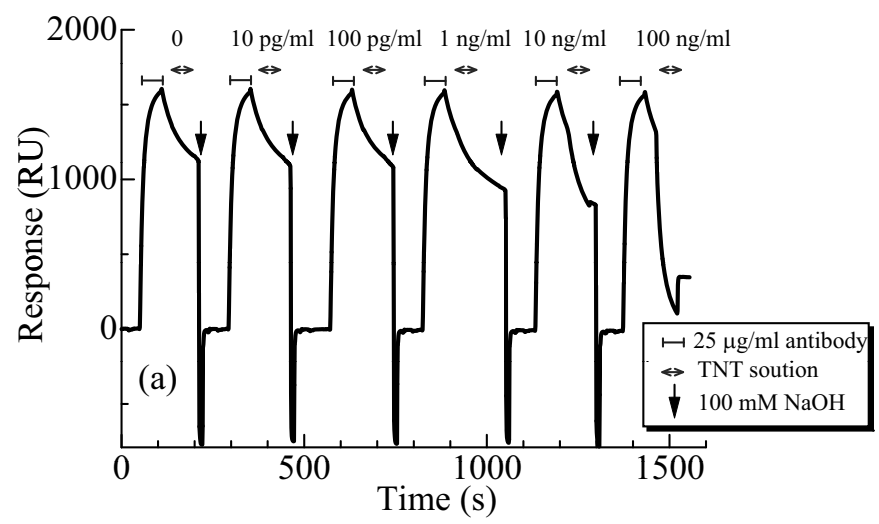

(a)

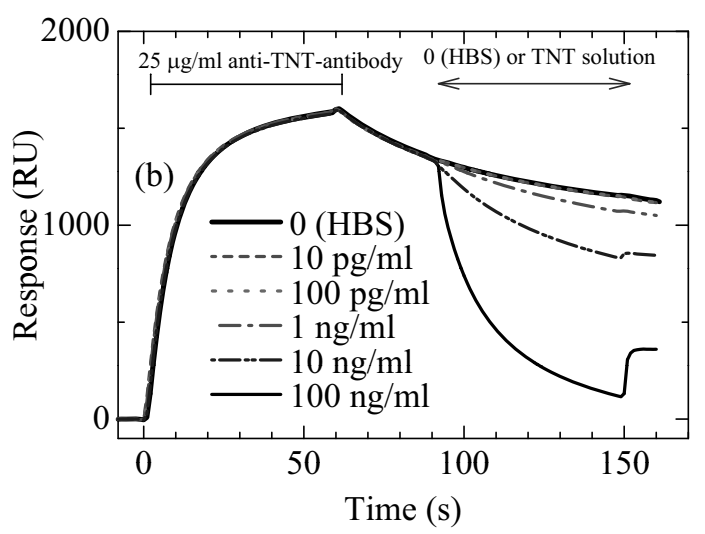

(b)

Fig. 2. (a) Sensorgrams of displacement immunosensor using $25 \mu \mathrm{g} / \mathrm{mL}$ TNT-Ab to detect various concentrations of TNT on DNP-gly-modified sensor chip. (b) Overlay of sensorgrams in (a).

ratio was higher than the DNP-gly-modified surface displacement ratio. The amount of antibody bound onto the DNP-aa-modified surface was lower than that on the DNP-glymodified surface. That is, the density of the antibody bound onto the DNP-aa-modified surface was lower than that of the antibody bound onto the DNP-gly-modified surface. When the antibody density is low, the molecular number ratio of the antibody to the antigen is favorable for displacement. In contrast, when the antibody density is high, the amount of the displaced antibody is large; however, in such a case, the displacement ratio is low. Similar results concerning the combination of a labeled antigen and an immobilized antibody have been reported. ${ }^{(9)}$ A lower immobilized antibody density provided a higher displacement efficiency in that study. Our TNT-analogue-immobilized surfaces showed the same trends; the DNP-aa-modified surface provided a higher displacement ratio at higher TNT concentrations. 


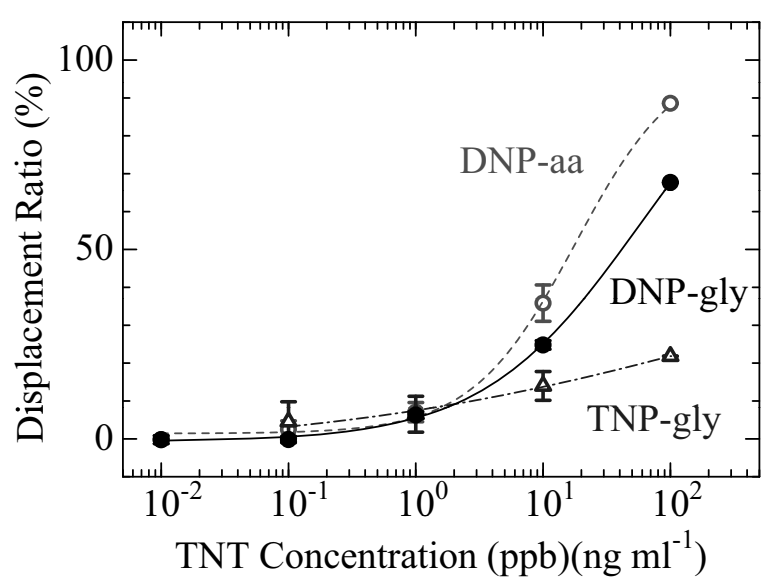

Fig. 3. Response curves obtained by displacement assay of three kinds of hapten-immobilized sensor surfaces.

Although the TNP-gly density of the TNP-gly-modified surface was comparatively low considering the amount of bound TNT-Ab, the displacement ratio was low at higher TNT concentrations. The affinity of TNP-gly for TNT-Ab was high, because TNP-gly was conjugated with keyhole limpet hemocyanin as an immunogen to produce TNT$A b{ }^{(20)}$ The affinity constant of the TNT-Ab- and TNP-gly-immobilized surfaces was 3.0 $\times 10^{7} \mathrm{M}^{-1}$, which is the highest among the three kinds of sensor surfaces. ${ }^{(4)}$ As shown in Fig. 3, the slope in the linear region of the TNT response curve for the DNP-aa-modified surface was the best among the three surface types.

Table 1 shows a summary of SDs at 1 ppb TNT, and 3 SDs and LODs for TNT on each sensor surface. The SD on the TNP-gly-modified surface was below $5 \%$, which is not large; however, the LOD was $45 \mathrm{ng} / \mathrm{mL}$ because the displacement ratio was low in a region of higher TNT concentration. The affinity constants between DNP-aa-TNT$\mathrm{Ab}$ and DNP-gly-TNT-Ab were $1.0 \times 10^{7} \mathrm{M}^{-1}$ and $2.0 \times 10^{7} \mathrm{M}^{-1}$, respectively. ${ }^{(4)}$ The DNP-aa-TNT-Ab affinity was slightly weaker than the DNP-gly-TNT-Ab affinity. We expected that the lowest LOD would be realized using the combination with the lowest affinity constant. Actually, the lowest LOD was achieved in the case of using the DNPgly-modified surface. It is probable that the SD for the DNP-gly-modified surface was slightly better than that for the DNP-aa-modified surface. The SD for the DNP-glymodified surface was reduced because the amount of antibody bound onto the sensor surface was one order of magnitude larger than that on the DNP-aa-modified surface, so the LOD obtained for the DNP-gly-modified surface was the lowest among the three kinds of surfaces.

The DNP-gly sensor chip showed the lowest TNT LOD among the three kinds of chips. Two more DNP-gly sensor chips were fabricated for determining overall LOD, considering the individual differences among sensor chips. The same experiments as 
Table 1

LODs were considered at 3 SDs for each TNT sensor chip.

\begin{tabular}{lccc}
\hline & SD $(\%)$ at $1 \mathrm{ng} / \mathrm{ml}(\mathrm{ppb})$ & 3 SDs $(\%)$ & LOD $(\mathrm{ng} / \mathrm{ml})(\mathrm{ppb})$ \\
\hline TNP-gly & 4.7 & 14.2 & 4.6 \\
DNP-aa & 2.5 & 7.6 & 1.4 \\
DNP-gly & 0.9 & 2.7 & 0.4 \\
DNP-gly (3 chips) & 1.8 & 5.5 & 0.9 \\
\hline
\end{tabular}

described above were conducted. TNT concentrations from $10 \mathrm{pg} / \mathrm{mL}$ (ppt) to $10 \mathrm{ng} / \mathrm{mL}$ (ppb) were measured on each DNP-gly chip, and the average displacement ratios and SDs for the three chips are shown in Fig. 4. The $10 \mathrm{pg} / \mathrm{mL}$ flows and $100 \mathrm{ng} / \mathrm{mL}$ flows were measured six and three times, respectively, and the other concentrations were measured nine times. The LOD was obtained by the same calculation in the case of Fig. 3, and the overall LOD was $0.9 \mathrm{ppb}$. Sub-ppb LODs levels were achieved with only $1 \mathrm{~min}$ flow of TNT solution. Flowing time of TNT solution longer than 1 min would enable lower LOD to be achieved.

In Fig. 4, the lower-TNT-concentration region is outside of the linear region for fitting. The displacement ratio of the lower-TNT-concentration region is low owing to a large amount of antibody binding to the reference. However, the amount of displaced TNT-Ab for $1 \mathrm{ng} / \mathrm{mL}$ TNT solution was observed to be about $94.2 \mathrm{RU}$ (average of 9 measurements on three DNP-gly sensor surfaces). This value is larger than the baseline noise $(0.6 \mathrm{RU})$ of the instrument, and indicates that an LOD of $0.9 \mathrm{ppb}$ is valid.

\subsection{Comparison of two measurement procedures}

Larsson et al. reported TNT concentration measurement by a displacement method based on SPR. ${ }^{21)}$ Oligo(ethylen glycol)-alkylthiols terminated with a hydroxyl group and a TNT analogue were synthesized and the sensor surface was modified with mixed SAMs using the synthesized chemicals. Their measurement procedure is different from our procedure. An anti-TNT antibody was bound to the sensor surface and spontaneous dissociation was allowed until it became stable. The amount of anti-TNT antibody dissociated following the sequential injections of 1,10 , and $100 \mathrm{ng} / \mathrm{mL}$ TNT solutions was measured. Dissociation was observed following the injection of $10 \mathrm{ng} / \mathrm{mL} \mathrm{TNT}$ solution. From the viewpoint of using the system in the field, the merit of Larsson et al.'s method is that the measurement of a reference value is not required, because the effect of baseline drift due to spontaneous dissociation was negligible. Its demerit is that about 1,500 $\mathrm{s}$ is required to reach a stable baseline.

We also tried to measure the amount of displacement in almost the same situation (data not shown). $50 \mu \mathrm{g} / \mathrm{mL}$ (ppm) TNT-Ab solution was allowed to flow over the DNP-glymodified sensor surface for $1 \mathrm{~min}$, and 2,400 s was required to reach a stable baseline. The maximum amount of bound TNT-Ab was about 1,100 RU at the end of flow; however, the amount was reduced to about $200 \mathrm{RU}$ by the time a stable baseline was attained. TNT solutions of various concentrations were sequentially injected without injection of the regeneration solution. RU reductions were observed following the 


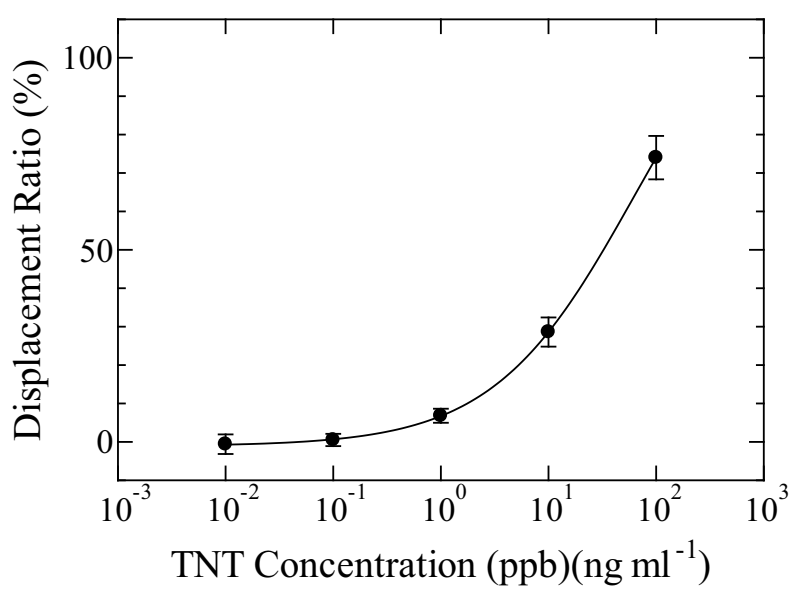

Fig. 4. Response curve for TNT using three DNP-gly-modified chips.

injection of $10 \mathrm{ng} / \mathrm{mL}$ and $100 \mathrm{ng} / \mathrm{mL}$ TNT solutions. However, there were no reductions following the injection the TNT solution less than $1 \mathrm{ng} / \mathrm{mL}$.

Antibodies are bivalent, and any antibody that binds to one hapten has a very high probability of binding to a second hapten. However, when the concentration of the antibody flowing over the surface is sufficiently high, it is considered that monovalent an antibody-hapten complex will form because of steric hindrance.(22) Namely, the ratio of monovalently bound antibody will increase. Immediately after the injection of an antibody is completed, monovalently bound antibodies (with weaker binding than bivalently bound antibodies) spontaneously dissociate from the surface. When the sensor response becomes stable, most of the remaining surface-bound antibodies are bivalently surface-bound antibodies. Therefore, a difference of one order of magnitude in the LOD was obtained using our developed measurement procedure.

\subsection{Detection of TNT using slope of sensorgram}

The slope of the sensorgram $10 \mathrm{~s}$ after the start of TNT injection was calculated to shorten the measurement time for detecting TNT. Measurement data obtained using the three DNP-gly chips described above were used in the analysis. We analyzed the data at $10 \mathrm{~s}$, because the sensorgram is little affected by differences in the refractive index of solvents or microvalve changes in the flow unit immediately after injection. The slope was calculated using a function of BIACORE X control software Ver. 2.2, ${ }^{(23)}$ which was fitted with a regression line using the least squares for time windows. The time window was set at $5 \mathrm{~s}$ (a point plus and minus $2 \mathrm{~s}$ ). Actually, $12 \mathrm{~s}$ is required for analysis after the start of TNT injection.

The ratio of the slope was calculated using

$$
R S=\left(\frac{S_{\mathrm{T}}}{S_{\mathrm{H}}}\right) \times 100(\%),
$$


where $S_{\mathrm{H}}$ is the slope for the reference HBS injection, and $S_{\mathrm{T}}$ is the slope for the TNT injection.

Figure 5 shows the response curves calculated using the slopes for the three DNPgly chips. The graph on the right side is an enlargement of the square area in the leftside graph. RS was high in the higher-TNT-concentration region. RS at $100 \mathrm{ppb}$ TNT, which was affected by the minimal difference in refractive index, as described above, was without offset. RS at 1 ppb TNT was $138.6 \%$ and the SD was 9.7\%. The LOD, which was calculated considering $3 \mathrm{SD}$, was about $0.7 \mathrm{ppb}$ at $130 \% \mathrm{RS}$. This LOD and detection time makes one-minute detection, including sampling with a wiper and the extraction process, possible.

\section{Conclusions}

We developed a measurement procedure using the displacement method and SPR to detect explosives and investigated the limit of TNT detection. In this procedure, TNT solutions were injected within $30 \mathrm{~s}$ after the end of anti-TNT antibody flow. That is, no waiting period was needed to reach a stable baseline for TNT solution injection.

We fabricated three kinds of sensor chips, on which TNT analogues were immobilized, namely, DNP-glycine, TNP-glycine, and DNP-acetic acid in ethyleneglycol-unit-containing SAMs. Commercially available TNT-Ab was used as the detector. The flow period of the TNT-Ab and TNT solutions was only $1 \mathrm{~min}$ at a flow rate of $10 \mu \mathrm{l} / \mathrm{min}$. An LOD of $0.4 \mathrm{ppb}$ was realized using the DNP-glycine-immobilized sensor surface. A final LOD of $0.9 \mathrm{ppb}$ was obtained from the results of measurements using the three different DNP-gly chips. Furthermore, the displacement immunosensor detected TNT with a $0.7 \mathrm{ppb}$ LOD within $12 \mathrm{~s}$ from the start of TNT injection, using the slope of the sensorgram. We will realize one-minute detection, including sampling with a wiper and the extraction process, using the displacement immunosensor.

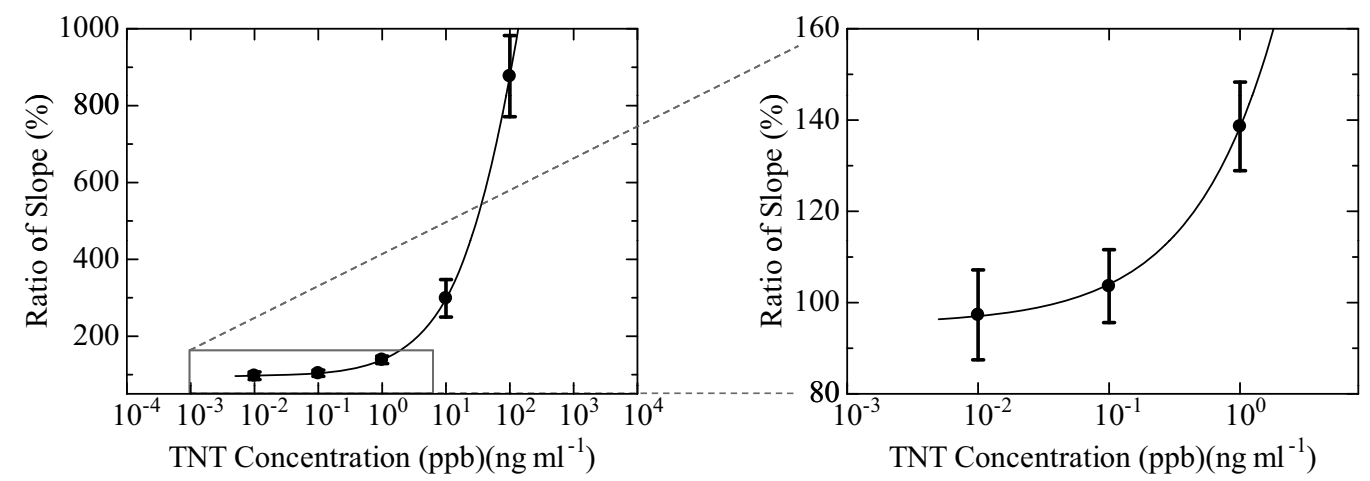

Fig. 5. Response curves for TNT calculated using sensorgram slopes. 


\section{Acknowledgments}

This work was partly supported by Japan Science and Technology Agency (JST) through Core Research for Evolutional Science and Technology (CREST). We would like to acknowledge Dr. J. Liang and Mr. K. Harada for their assistance in our study.

\section{References}

1 T. Onodera, N. Miura, K. Matsumoto and K. Toko: Anti-Personnel Landmine Detection for Humanitarian Demining, K. Furuta and J. Ishikawa (ed.) (Springer, London, 2009) pp. 193205.

2 K. Matsumoto, A. Torimaru, S. Ishitobi, T. Sakai, H. Ishikawa, K. Toko, N. Miura and T. Imato: Talanta 68 (2005) 305.

3 K. Nagatomo, K. Matsumoto, K. Toko and N. Miura: J. Fac. Agr. Kyushu Univ. 54 (2009) 173.

4 Y. Mizuta, T. Onodera, P. Singh, K. Matsumoto, N. Miura and K. Toko: Biosens. Bioelectron. 24 (2008) 191.

5 K. Nagatomo, T. Kawaguchi, N. Miura, K. Toko and K. Matsumoto: Talanta 79 (2009) 1142.

6 P. Singh, T. Onodera, Y. Mizuta, K. Matsumoto, N. Miura and K. Toko: Sens. Actuators, B 137 (2009) 403.

7 G. A. Wemhoff, S. Y. Rabbany, A. W. Kusterbeck, R. A. Ogert, R. Bredehorst and F. S. Ligler: J. Immunol. Methods 156 (1992) 223.

8 A. W. Kusterbeck, G. A. Wemhoff, P. T. Charles, D. A. Yeager, R. Bredehorst, C. Vogel and F. S. Ligler: J. Immunol. Methods 135 (1990) 191.

9 S. Y. Rabbany, A. W. Kusterbeck, R. Bredehorst and F. S. Ligler: J. Immunol. Methods 168 (1994) 227.

10 T. M. Green, P. T. Charles and G. P. Anderson: Anal. Biochem. 310 (2002) 36.

11 G. P. Anderson, S. C. Moreira, P. T. Charles, I. L. Medintz, E. R. Goldman, M. Zeinali and C. R. Taitt: Anal. Chem. 78 (2006) 2279.

12 T. Onodera, J. Liang, K. Miyahara, K. Matsumoto, N. Miura, T. Imato and K. Toko: Chemical Sensors 20 Supplement B (2004) 100.

13 J. Nakamura: Explosion 15 (2005) 134 (in Japanese).

14 T. Onodera, T. Shimizu, N. Miura, K. Matsumoto and K. Toko: IEEJ Trans. SM. 130 (2010) 269.

15 B. Liedberg, C. Nylander and I. Lundstrom: Biosens. Bioelectron. 10 (1995) i.

16 S. Y. Rabbany, W. J. Lane, W. A. Marganski, A. W. Kusterbeck and F. S Ligler: J. Immunol. Methods 246 (2000) 69.

17 U. Narang, P. R. Gauger and F. S. Ligler: Anal. Chem. 69 (1997) 2779.

18 W. A. Kaptein, J. Korf, S. Cheng, M. Yang, J. F. C. Glatz and R. Renneberg: J. Immunol. Methods 217 (1998) 103.

19 E. Stenberg, B. Persson, H. Roos and C. Urbaniczky: J. Colloid Interface Sci. 143 (1991) 513.

20 A. Zeck, M. G. Weller and R. Niessner: J. Anal. Chem. 364 (1999) 113.

21 A. Larsson, J. Angbrant, J. Ekeroth, P. Mansson and B. Liedberg: Sens. Actuators, B 113 (2006) 730 .

22 M. Oda and T. Azuma: Molecular Immunology 37 (2000) 1111.

23 Biacore, A. B., BIACOR X Instrument Handbook (version AA), (Uppsala, Sweden, 1997) p. D-23. 


\section{About the Authors}

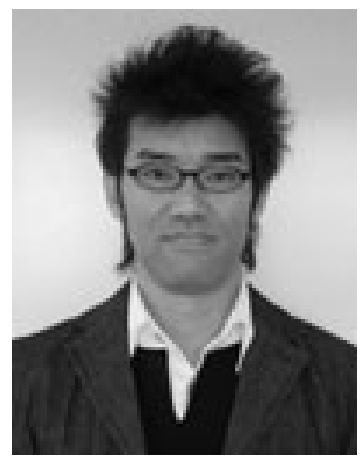

Takeshi Onodera received his BA degree from Toyama University of International Studies, Toyama, Japan, in 1996. He received his MEd degree in technology education and PhD degree in engineering from Kanazawa University in 1998 and 2001, respectively. He is now an assistant professor at Kyushu University. His main research interest is the development of biosensors for safety and security. He is a member of The Institute of Electrical Engineers of Japan, the Electrochemical Society of Japan and the Japan Explosives Society.

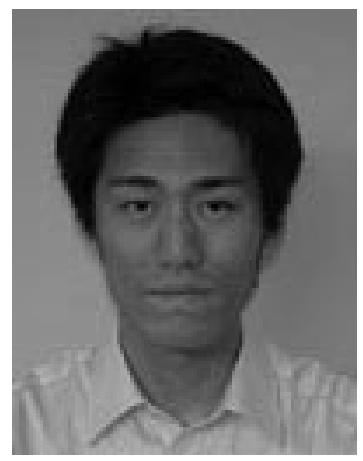

Yutaka Mizuta received his BSc degree in organic chemistry from Waseda University, Japan in 2000. He received his $\mathrm{PhD}$ degree in electrical engineering from Kyushu University, Japan in 2010. He works for Central Customs Laboratory, Japan. He is a member of the Institute of Electrical Engineers of Japan.

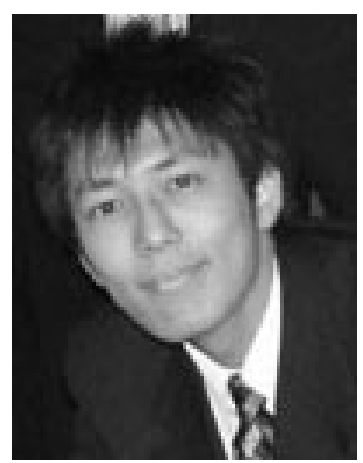

Kazuhiko Horikawa received his $\mathrm{BE}$ and $\mathrm{ME}$ in electrical engineering, from Kyushu University, Japan, in 2006 and 2008, respectively. 

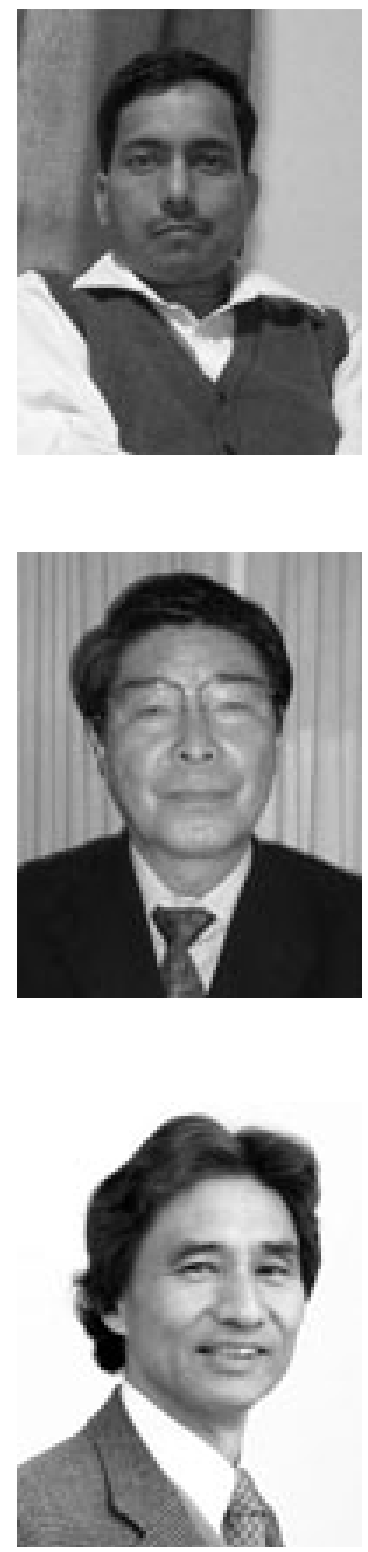

Praveen Singh received his Ph.D.(Physics) from Indian Institute of Technology Delhi, New Delhi, India, in 2001. He worked as a scientist at CAZRI Jodhpur, India. Presently, he is working at Indian Veterinary Research Institute Izatnagar, Bareilly, UP, India, as a senior scientist. He was a JSPS Fellow at Professor Kiyoshi Toko's laboratory, Department of Electronics, Kyushu University, from 2005-2007. He is a member of Indian Biophysical Society and Indian JSPS alumni association. His research interests include radar absorbing materials, magnetic nanomaterials and optical biosensors for explosive and biological molecules.

Kiyoshi Matsumoto is a professor at Sojo University. He had been a professor at Kyushu University since 1989, and retired in 2010. He received his DAgr degree from the Department of Food Science and Technology, Kyushu University, Japan. He has published more than 200 original papers and 80 reviews. His current research is concentrated on the development of enzyme sensors for food quality control and the preparation of new antibodies for the development of SPR biosensors.

Norio Miura has been a professor at Kyushu University since 1999. He received his DEng degree in 1980 from the Department of Applied Chemistry, Kyushu University, Japan. He has published more than 400 original papers and has nearly 120 patents. His current research is concentrated on the development of high-performance chemical sensors and other electrochemical functional devices. 


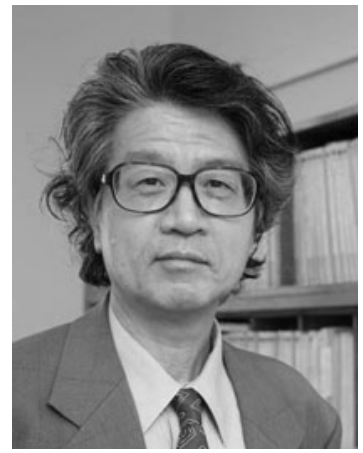

Kiyoshi Toko received his $\mathrm{BE}, \mathrm{ME}$ and $\mathrm{PhD}$ degrees in electrical engineering from Kyushu University in 1975, 1977 and 1982, respectively. He is now a distinguished professor and dean of the Graduate School of Information Science and Electrical Engineering of Kyushu University. He is a member of the Japan Society of Applied Physics, the Institute of Electrical Engineers of Japan and the Japanese Association for the Study of Taste and Smell. 